EPJ Web of Conferences 43, 04005 (2013)

DOI: $10.1051 /$ epjconf/20134304005

(C) Owned by the authors, published by EDP Sciences, 2013

\title{
Asteroseismology of hot B subdwarf stars
}

\author{
S. Charpinet ${ }^{1, a}$, V. Van Grootel $^{2}$, P. Brassard ${ }^{3}$, G. Fontaine ${ }^{3}$, E.M. Green ${ }^{4}$ \\ and S.K. Randall ${ }^{5}$ \\ ${ }^{1}$ CNRS, Université de Toulouse, UPS-OMP, IRAP \\ ${ }^{2}$ Institut d'Astrophysique et de Géophysique, Université de Liège \\ ${ }^{3}$ Département de Physique, Université de Montréal \\ ${ }^{4}$ Steward Observatory, University of Arizona \\ ${ }^{5}$ European Southern Observatory, Garching
}

\begin{abstract}
Non-radial pulsations in Extreme Horizontal Branch stars (also known as hot B subdwarfs or sdB stars) offer strong opportunities to study, through asteroseismology, the structure and internal dynamics of stars in this intermediate stage of stellar evolution. Most sdB stars directly descend from former red giants and are expected to evolve straight into white dwarfs after core helium exhaustion. They thus represent the most direct link between these two stages. Their properties should therefore reflect both the outcome of the core evolution of red giant stars and the initial state for a fraction of the white dwarfs. We review the status of this field after a decade of efforts to exploit both p-mode and g-mode pulsating sdB stars as asteroseismic laboratories. From the discoveries of these two classes of pulsators in 1997 and 2003, respectively, up to the current epoch of data gathering of unprecedented quality from space, a lot of progress has been made in this area and prospects for future achievements look very promising.
\end{abstract}

\section{INTRODUCTION}

Most hot B subdwarf (sdB) stars (see [1] for a complete review) are believed to be former red giant stars that have lost all but a tiny fraction of their H-rich envelope during or just before the onset of helium burning in the core. These stars settle down on the so-called Extreme Horizontal Branch (EHB), where they quietly consume their helium during $\sim 10^{8}$ years. Hot B subdwarfs are hot $\left(T_{\text {eff }}=22,000-40,000 \mathrm{~K}\right)$ and compact $(\log g=5.1-6.2)$ objects with a mass distribution strongly peaked near $\sim 0.47 M_{\odot}$ [2], while their radii extend from 0.10 to $0.25 R_{\odot}$, typically. The helium cores of sdB stars are still surrounded by a very thin H-rich envelope, residual of the former envelope of the star on the red giant branch, that is at most $\sim 1 \%$ of the total stellar mass. Hot subdwarfs then evolve directly to the white dwarf stage without experiencing another red giant phase (the AGB phase) as most other horizontal branch stars do after core helium exhaustion. It is estimated that $\sim 2 \%$ of the white dwarfs come from this channel [3].

A significant fraction of the $\mathrm{sdB}$ stars shows non-radial oscillations, thus opening up the way for detailed asteroseismic investigations. They come in two groups (see Fig. 1) featuring either short period (1-10 mn) variations due to low-order, low-degree acoustic waves ( $p$-modes; the V361 Hya stars discovered in 1997 [4]) or long period $(\sim 1-4 \mathrm{~h})$ modulations due to mid/high order, low degree gravity waves ( $g$-modes; the V1093 Her stars discovered in 2003 [5]). Some sdB stars, called hybrid pulsators, belong to both classes as they show modulations due to both p- and g-modes [6].

\footnotetext{
ae-mail: stephane.charpinet@irap.omp.eu
}

This is an Open Access article distributed under the terms of the Creative Commons Attribution License 2.0, which permits unrestricted use, distribution, and reproduction in any medium, provided the original work is properly cited. 
EPJ Web of Conferences

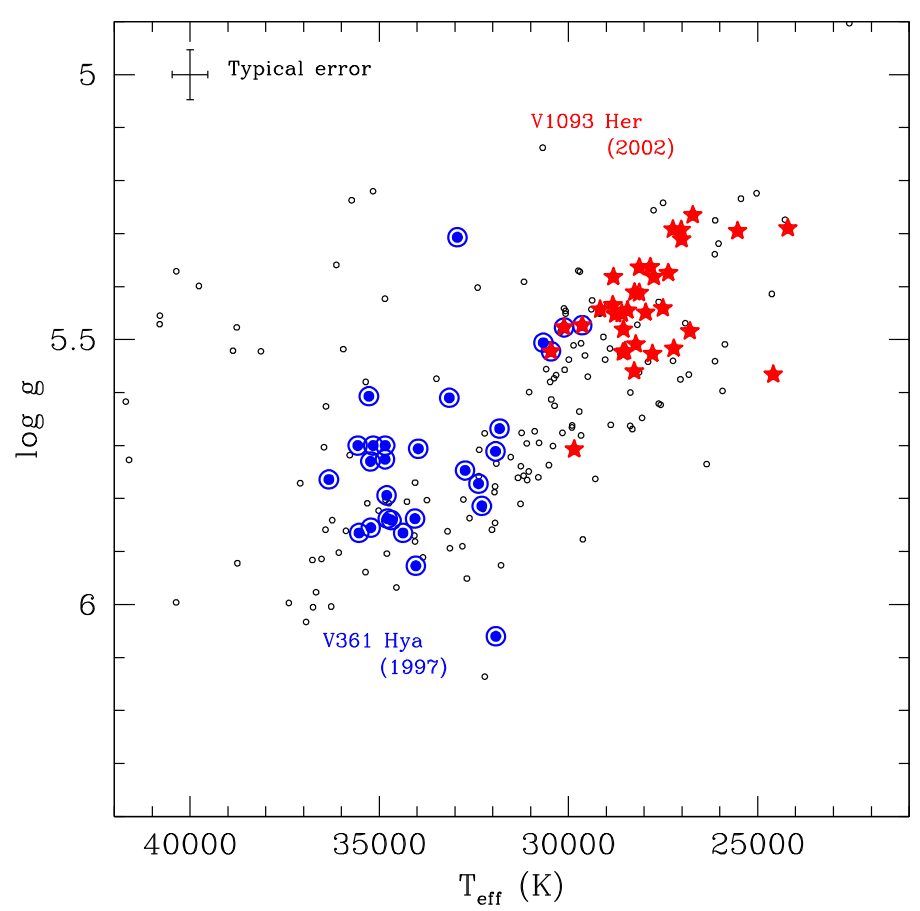

Figure 1. Hot B subwarf pulsators in the $\log g-T_{\text {eff }}$ diagram. The short period, $p$-mode pulsators of the V361 Hya type (filled circles with an annulus) are found in the high temperature range while the long period, V1093 Her type $g$-mode pulsators (filled star symbols) occupy the low temperature region. The two instability strips have some overlap where hybrid pulsators (filled stars surrounded by an annulus) can be found.

The mechanism driving the oscillations is well identified. It is the same $\kappa$-effect for both classes that involves partial ionization of heavy metals (primarily iron) in the opacity bump ( $Z$-bump) region. Radiative levitation is however playing a fundamental role in making this mechanism effective enough to generate the observed oscillations $[7,8]$.

Important observational efforts to detect and characterize the oscillations in sdB stars have been made during the past decade. These include surveys to find the pulsators $[4,9,10]$, dedicated groundbased white light photometric campaigns to provide key data for asteroseismology [11, 12] (see Fig. 2), attempts to identify modes through multicolor photometry [13-15] and time resolved spectroscopy $[16,17]$, and most recently ultra high precision photometry from space with CoRoT [18] and Kepler $[19,20]$ (see Fig. 2). These efforts have permitted major breakthroughs in investigating the properties of these stars with the tools of asteroseismology. We discuss some of them in the following sections.

\section{MODE PROPERTIES IN SDB STARS}

Asteroseismic inferences of the global parameters of sdB stars reflect the dependency of the pulsation spectrum to the internal structure. It is therefore important to explore how the oscillation modes behave in these stars in order to understand the type of constraints that asteroseismology can provide. Mode properties in sdB stars have been extensively described in [21-23], although, along with the $p$-modes, only the low-order g-modes had been investigated at that time, since the g-mode pulsators had not yet been discovered. We extend here these studies, focusing in particular on the $g$-mode spectrum that is being revealed in much greater details than before by current space observations with CoRoT and Kepler. 
Ageing Low Mass Stars: From Red Giants to White Dwarfs
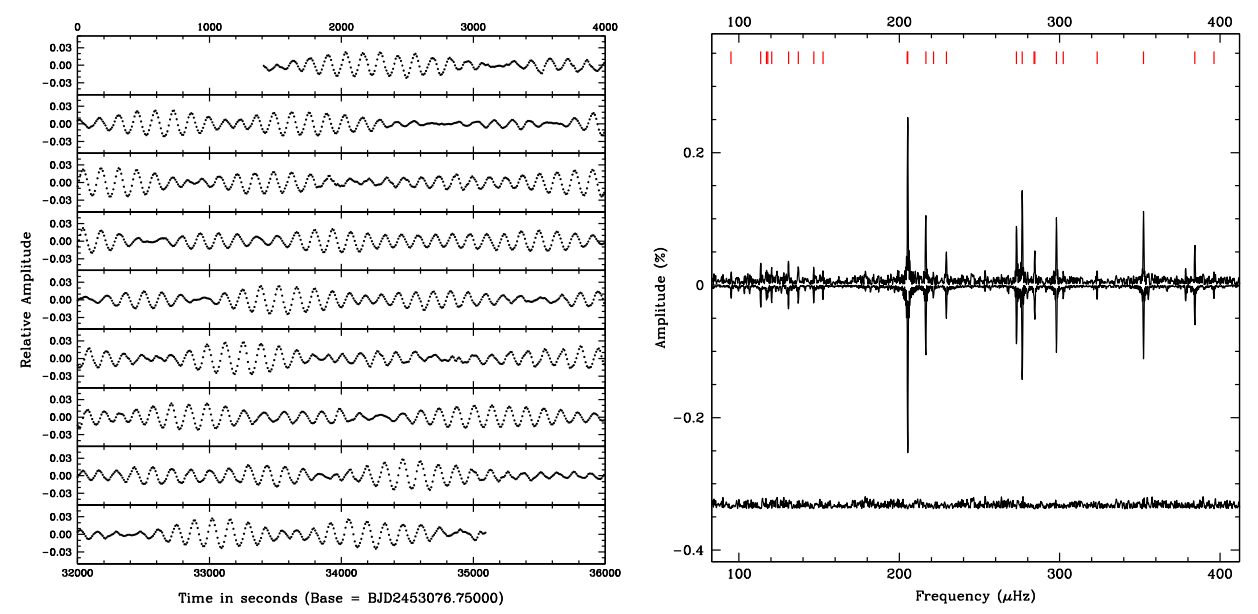

Figure 2. Left panel: Example of a light curve from a p-mode sdB pulsator (PG 1219+534) observed in 2004 at the Canada-France-Hawaii Telescope with the fast-photometer LAPOUne (from [12]). Right panel: Example of $g$ modes detected in KPD 1943+4058 after one month of Kepler observations. The spectrum upside-down is the signal reconstructed from the extracted frequencies. The lower curve is the residual in Fourier space after subtraction of the reconstructed light curve in the time domain.

Figure 3 shows a typical propagation diagram from a hot B subdwarf model. The angular frequencies shown $(\sigma=2 \pi \nu=2 \pi / P)$ cover the full range of modes observed in these stars. This includes the loworder $p$-modes from $k=1$ to $k=10$ that are representative of the acoustic waves detected in the short period pulsators of the V361 Hya type. At lower frequencies, it includes the $g$-modes up to radial order $k=70$, thus covering the range of modes detected with Kepler in V1093 Her stars. One can see from this diagram that the observed $p$-modes are envelope modes that are reflected back to the surface well before reaching the core of the star. In contrast, the $g$-modes propagate much deeper, down to the limit of the convective core and, especially for the highest order modes, quite high in the envelope. Gravity modes clearly probe a much larger part of the star than the $p$-modes do. The latter in particular will hardly be sensitive to the structure of the innermost region of the star.

Two prominent spikes in the Brunt-Väisälä (BV) frequency profile are the signatures of the steep chemical transitions that occur between the H-rich envelope and the He mantle (near $\log q=-3.0$ ) and, much deeper, between the $\mathrm{C}-\mathrm{O}$ enriched mixed (convective) core and the base of the He mantle. These steep transitions are known to interfere with the pulsations by producing partial wave reflections. The modes having nodes (the filled circles shown in Fig. 3) that fall close to these transitions can either be partially trapped above or confined below the transition. Clearly from Fig. 3, the outermost $\mathrm{He} / \mathrm{H}$ transition between the envelope and the mantle will produce such trapping effects on both $p$ and $g$-modes. In particular, depending on the thickness of the envelope, the associated spike in the BV frequency migrates (between $\log q \sim-2.0$ and $\log q \sim-5.0$ for typical sdB stars), affecting differently the oscillation spectrum. The deeper $\mathrm{C}-\mathrm{O} / \mathrm{He}$ transition between the mixed core and the mantle (at $\log q \sim-0.20$ ) is out of reach for the $p$-modes, which therefore have no sensitivity to it. In contrast, the $g$-modes have nodes in that area indicating that trapping effects from this chemical transition will also occur. In particular, the size of the mixed core, i.e., the position of the spike in the BV frequency (typically between $\log q \sim-0.15$ and $\log q \sim-0.40$ ), will have an imprint on the $g$-mode period spectrum.

Figure 4 shows the signatures on the $g$-mode period spectrum induced by the above mentioned chemical transitions, depending on their respective position inside the star. Both the $\mathrm{He} / \mathrm{H}$ and $\mathrm{C}-\mathrm{O} / \mathrm{He}$ transitions produce distinct patterns of trapping structures showing up as cyclic perturbations of the 


\section{EPJ Web of Conferences}

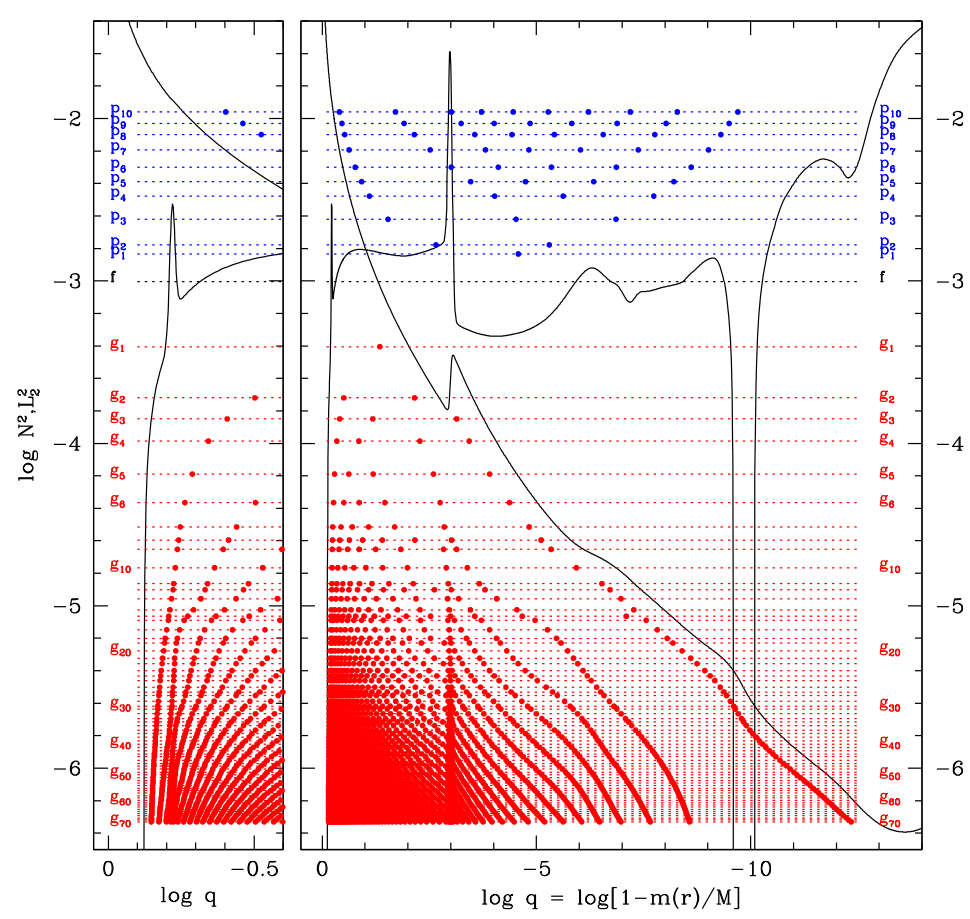

Figure 3. Propagation diagram for modes of degree $\ell=2$ in a representative sdB model. The region where the mode angular frequencies exceed both the Lamb $\left(L_{2}\right)$ and Brunt-Väisälä $(N)$ frequencies corresponds to the acoustic cavity where $p$-modes propagate ( $p$-modes up to $k=10$ are shown). The g-mode cavity is found in the region where the angular frequency is lower than $L_{2}$ and $N$. Gravity modes with radial orders up to $k=70$ are shown. Each filled circle indicates the position of a node in the radial displacement eigenfunction. The left panel is a close-up view of the central region of the $\operatorname{star}(\log q=0$ being the center $)$.

mean period spacing between consecutive modes of the same degree $\ell$. It is these dependencies that allow us to extract information on both the size of the H-rich envelope and the size of the mixed core with asteroseismology using the g-modes observed in several sdB pulsators monitored from space (see next Section).

From the left panel of Fig. 4, it is interesting to note, especially for the models with the thickest envelopes $(\log q \gtrsim-3.5)$ representative of the cooler long period pulsators, that the trapping patterns are not uniform in amplitude in the whole period range. The trapping structures tend to disappear for the higher order modes (with long periods / low frequencies). This fact is particularly important for the interpretation of the rich g-mode spectra that can be extracted from the Kepler observations.

To discuss further this point, we examine a typical evolutionary model of a hot B subdwarf star having a relatively thick envelope, $\log \left(M_{\text {env }} / M_{\text {tot }}\right) \sim-2.4$, of initial solar composition. The model is slightly evolved away the ZAEHB and the evolutionary calculations include, in particular, helium settling that affects the steepness of the $\mathrm{He} / \mathrm{H}$ transition profile as well as the composition of the envelope (Fig. 5 provides basic information on the chemical stratification and structure of the star). Figure 6 shows, for this representative model, the corresponding $g$-mode distributions in two different ways: first, in terms of period spacings (like in Fig. 4) and second, as echelle diagrams. Clearly, nearly constant period spacings at high radial orders (long periods) are observed, translating in the formation of a wavy ridge in the echelle diagram. However, below $5000 \mathrm{~s}$ (for lower order modes), this spacing is essentially lost due to more prominent trapping structures and the modes are seemingly distributed randomly in the echelle diagram. It is enlightening to qualitatively compare this theoretical plots to 
Ageing Low Mass Stars: From Red Giants to White Dwarfs
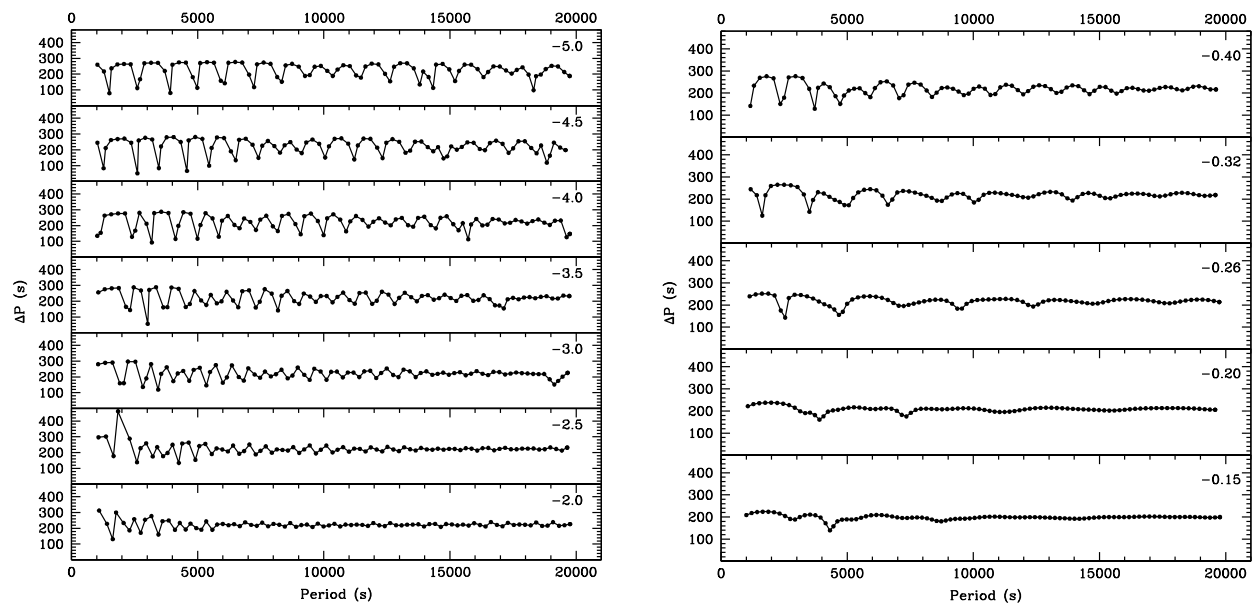

Figure 4. Gravity mode trapping structures in $\mathrm{sdB}$ stars. The left panel shows the period spacings $(\Delta P)$ of consecutive $g$-modes $(\Delta k=1)$ as a function of the period for a series of models on the ZAEHB with varying H-rich envelope mass, i.e. $\log q(H)=\log \left(M_{\text {env }} / M_{\text {tot }}\right.$ ) from -5.0 (thin envelope; top) to -2.0 (thick envelope; bottom). The right panel shows the period spacings for a series of models where the contribution of the $\mathrm{He} / \mathrm{H}$ mantle/envelope chemical transition has been removed, leaving only the signature of the $\mathrm{C}-\mathrm{O} / \mathrm{He}$ transition at the mixed core boundary whose position, $\log q$ (core) $=\log \left(1-\mathrm{M}_{\text {core }} / \mathrm{M}_{\mathrm{tot}}\right)$, varies from -0.40 (large mixed core; top) to -0.15 (small mixed core; bottom).

observational echelle diagrams produced recently for $g$-mode sdB pulsators based on Kepler data (e.g, Figure 6 in [24]). Both show some striking similarities. We point out however that the model used in this comparison is a chemically stratified model and we stress that care must be taken in interpreting these observed period spacings. Contrary to the interpretation of Reed et al. [25] that these stars should be much less stratified than expected because of the occurrence of nearly constant period spacings, we show here that the situation is more complex and that the observations are in fact consistent with typical, fully stratified, sdB models that also show nearly constant period spacings for the higher order $g$-modes at low frequencies.

\section{ASTEROSEISMOLOGY OF SDB STARS: STRUCTURAL PARAMETERS}

Detailed quantitative seismic investigations of hot B subdwarf stars are among the pioneering efforts that pulled the field of asteroseismology closer to its full potential. It started with the work of Brassard et al. [26] on the $p$-mode sdB pulsator PG 0014-067. Since then, the technique has been constantly refined and applied to $15 \mathrm{sdB}$ pulsators so far (see [2] for a list of these analyses with the associated references). The most recent results in this area, largely triggered by new ultra high precision photometry from space with CoRoT and Kepler, are detailed seismic inferences obtained for the first time with $g$-modes, leading to completely new constraints on the internal structure of these stars, in particular on their core properties (see below).

The technique developed for these seismic investigations is based on the so-called forward modeling method. We stress that it is so far the only approach, at least for sdB pulsators, that has proven capable of objectively deriving quantitative information on the stars themselves. It consists of matching theoretical oscillation spectra computed with appropriate stellar models to the observed frequencies of a given star. By finding the best possible match(es) in the model parameter space, some of the main structural parameters of the star can be derived. As a comparison, we point out that this approach is similar in essence to the method of deriving atmospheric parameters of stars by fitting line profiles computed from appropriate model atmospheres to the observed spectra. 

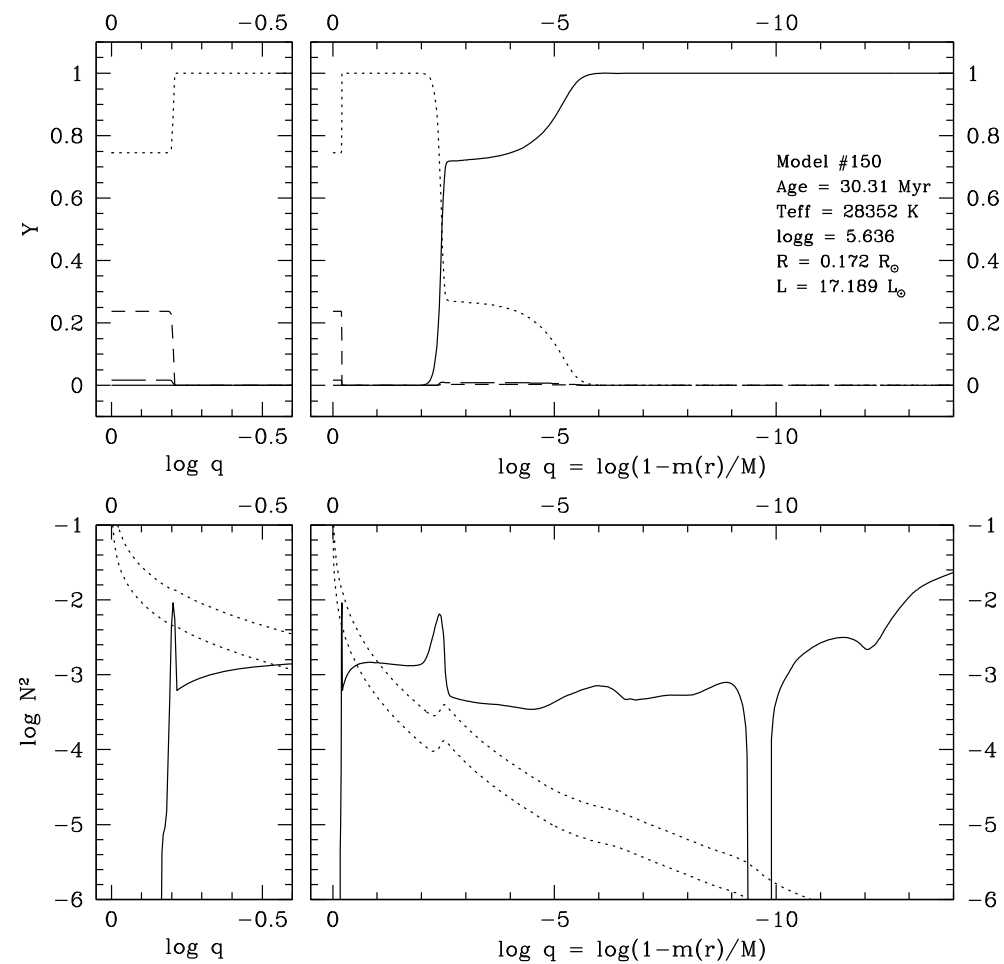

Figure 5. Chemical stratification in a representative evolutionary model of a hot B subdwarf star (upper panel). The mass fractions of hydrogen (plain curve), helium (dotted curve), carbon (dashed curve), and oxygen (longdashed curve) are shown as a function of the logarithmic fractional mass depth $\log q=\log (1-m(r) / M)$. The corresponding profiles for the Brunt-Väisälä frequency ( $N$; plain curve) and $\ell=1, \ell=2$ Lamb frequencies (dotted curves) are represented in the lower panel. Left subpanels show close-up views of the central region of the star.

Different types of models can be used to probe hot B subdwarf pulsators with asteroseismology. We argue that static structures (as opposed to evolutionary models) are the most effective tools to achieve this objective. Mode frequencies are not directly sensitive to stellar evolution (because the pulsation periods are generally much shorter than the evolutionary timescale). Hence, the modes just "feel" the instantaneous structure of the star (density, chemical stratification, ...) at a given time. This structure can be more easily parameterized with static models in order to explore more exhaustively various configurations. Evolutionary models are more tightly bound to physical processes that shape, over time, the star internal structure. However, while this may appear more physically sound, one should never forget that these processes and the past history of stars are generally not free of uncertainties that tend to accumulate as evolution proceeds (see the contribution of A. Weiss in these proceedings). In other words, what we seek to achieve by using static stellar models for quantitative asteroseismic studies is to find the instantaneous (static) structure of the star that best reproduces the observed pulsation properties (and other constraints). The question of how, with evolutionary processes, a star can then reach the seismically constrained structure is yet another independent step.

For asteroseismological purposes, we first developed static envelope models (also called 2nd generation models [27]) assuming constant luminosity inside the star, which is a very good approximation for extreme horizontal branch stars where the helium burning energy production is strongly confined around the center of the star and where no significant hydrogen shell burning occurs at the base of the envelope. A key ingredient in these models was the inclusion of radiative levitation on iron under the assumption of diffusive equilibrium. These models are most suitable for detailed 
Ageing Low Mass Stars: From Red Giants to White Dwarfs

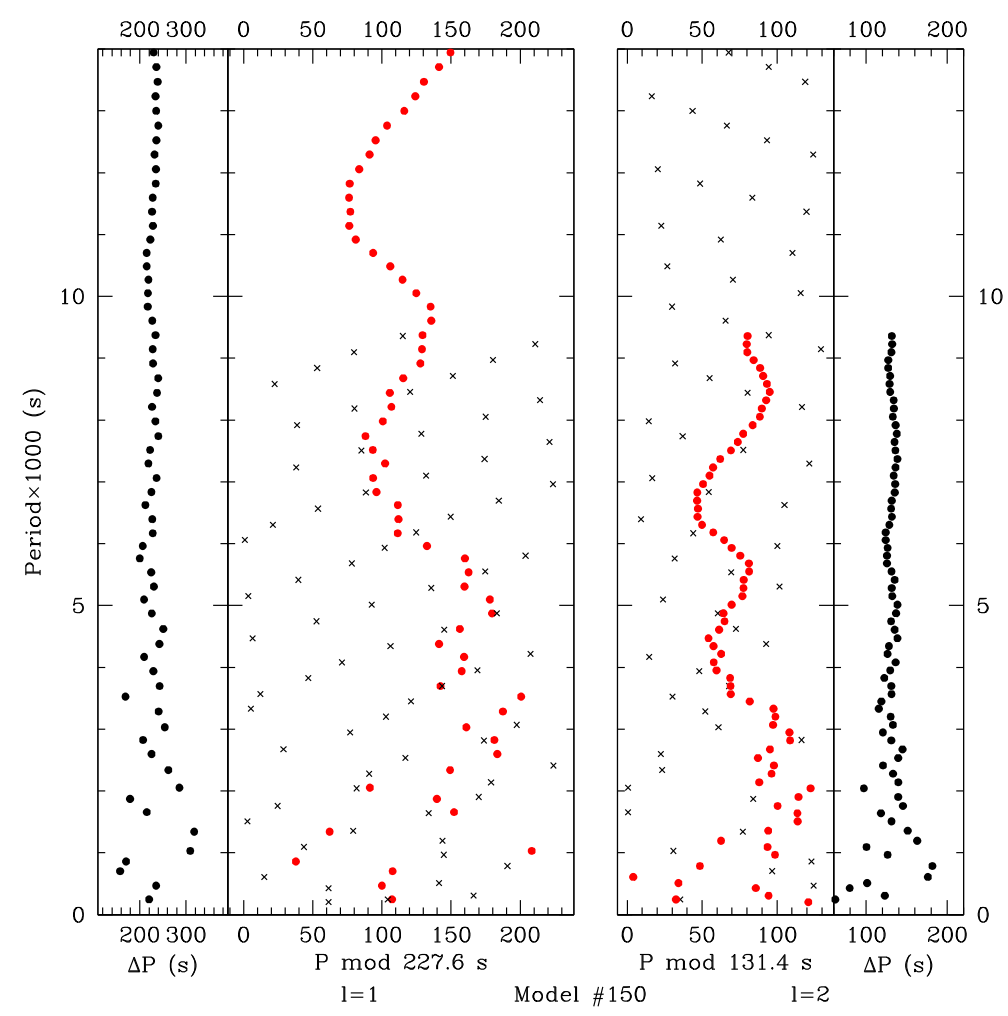

Figure 6. Theoretical echelle diagram for g-modes up to $k=70$ in the representative evolutionary model. For the $\ell=1$ (left panels) and $\ell=2$ (right panels) series, the period spacing $(\Delta P)$ of consecutive modes (with $\Delta k=1)$ is shown as a function of the period $(P)$ along with the corresponding echelle diagram representing the period modulo the mean period spacing (which depends on $\ell$ ) vs the period of the modes. The filled circles indicate modes of a given degree $(\ell=1$ for the left panels and $\ell=2$ for the right panels). The crosses show modes belonging to the other series $(\ell=2$ for the left panel and $\ell=1$ for the right panel).

studies of the $p$-mode sdB pulsators because acoustic modes are envelope modes that are not affected by the detailed structure of the deepest regions of the star. The periods (frequencies) of these modes are accurately calculated on the basis of these models. The 2 nd generation models proved themselves quite successful for deriving, at a very interesting level of precision, some of the fundamental parameters of the majority of the sdB pulsators analyzed so far (see [2]). In particular, one star, the compact eclipsing sdB+dM binary system PG 1336-018, provided a fundamental test of the method and of the models themselves by allowing us to cross-check some of the seismically derived quantities (in particular, the mass and radius) with completely independent estimations derived from the orbital lightcurve analysis [28]. This test showed a clear convergence (within the 1 sigma errors of each technique) of the derived values for $\log g, M$, and $R$, thus demonstrating that, at least for these quantities, the asteroseismic inferences are precise and accurate. This is particularly important for assessing the reliability of asteroseismic mass determinations that are (and will be) essential to clarify which channels are relevant for the formation of sdB stars ([2] and see V. Van Grootel et al. in these proceedings).

The latest breakthrough in hot B subdwarf asteroseismology has come with the exploitation of the g-mode pulsations observed in the V1093 Her stars. Second generation envelope models are no longer suitable to compute precise gravity mode frequencies because these modes are too sensitive to the deepest regions of the star. A third generation of complete static models was then developed to accurately handle $g$-modes [29]. These models are computed assuming thermal equilibrium for a given 
chemical stratification and core composition. Nuclear reaction rates are calculated to evaluate the energy balance but the structure is not evolved in time. As in the case of the 2 nd generation models, radiative levitation in equilibrium with gravitational settling of iron is included in these newer models.

So far, three $g$-mode sdB pulsators monitored by CoRoT (KPD 0629-0016 [18, 30]) and Kepler (KPD 1943+4058 [31]; KIC 02697388 [32]) have been analyzed in detail using the 3rd generation models. These seismic investigations led to the determination of key parameters of these stars, including in particular new constraints on the size of the mixed core. This is a direct consequence of the sensitivity of $g$-modes to the chemical transition between the $\mathrm{C}-\mathrm{O}$ enriched mixed core and the surrounding helium mantle, as discussed in the previous section. It is found from these analyses that the mixed core in sdB stars is somewhat larger than expected from typical evolutionary models that consider only convection (and semi-convection at a later age of the evolution) as a mixing process occurring in these cores. This suggests that other mixing mechanisms, including overshooting and possibly effects like turbulent rotational mixing in case of differential rotation, may be at work at the core boundary in these stars.

Deep asteroseismic probing of the core properties of extreme horizontal branch stars is one of the new very promising possibilities offered by $g$-modes. This will help shed light and bring strong constraints on physical mechanisms that are known to be important in stars, but still poorly understood. A dozen of sdB stars monitored with Kepler are still waiting to be scrutinized with this technique and will undoubtedly allow us to progress on this front.

\section{ASTEROSEISMOLOGY OF SDB STARS: ROTATION}

Another promising possibility with asteroseismology is to measure the internal dynamical properties of extreme horizontal branch stars. This can be done by exploiting the signature of rotation in observed oscillation spectra of sdB stars. Rotation lifts the $2 \ell+1$ degeneracy of mode frequencies and produce multiplet structures of equidistant spacings in frequency (at first order) for a given mode of degree $\ell$ and radial order $k$, provided that the rotation is not too fast.

Seismic inferences of rotation in sdB stars have remained relatively few (but see [28]). This is most likely due, in retrospect, to the fact that most of these stars are likely very slow rotators that cannot be resolved, in terms of the seismic signature of rotation, with the typical duration of ground-based monitoring campaigns. The situation is currently changing, in particular with the very long time baseline offered by the Kepler data. A distinction needs to be made between the two following situations.

For the pulsating sdB stars that are found in close binary systems, the rotation of the star may be strongly affected by tidal effects. So far, seismic information on sdB stars in such systems have been obtained for 5 objects. PG 1336-018, a sdB pulsator with a close dM companion, has been shown to have a rotation synchronized at the orbital period of $2.42 \mathrm{~h}$. Moreover, in that case, the seismic data strongly suggest that the synchronization has been achieved down to approximately half the radius of the star. Below that limit, the observed $p$-modes quickly lose their sounding power and the rotation can no longer be constrained [28]. With Kepler, cases of non-synchronized sdB stars with close companions have emerged. KIC11179657 and KIC02991403 both have a dM companion with an orbital period of $\sim 9.6 \mathrm{~h}$ while clear seismic rotational signatures suggest that these stars rotate with a period of $\sim 7.4 \mathrm{~d}$ and $\sim 10.3 \mathrm{~d}$, respectively [33]. The star B4 in the cluster NGC 6791 also appears to be unsynchronized with a rotation period estimated at $\sim 9.6 \mathrm{~d}$ while the orbital period is $\sim 9.4 \mathrm{~h}$ [34]. The finding of both synchronized and unsynchronized systems among the sdB stars in close binaries is a very interesting situation for testing tidal synchronization timescales and theories, especially as detailed asteroseismology can potentially provide age estimates (since the onset of helium burning in the core) for the sdB component.

The second situation is when the $\mathrm{sdB}$ star is an isolated object with no apparent companion. The rotation status of these stars should then be the product of secular evolution and be directly related to their past history. Among the isolated sdB pulsators, seismic estimates of the rotation rate exist for only 4 stars: $\sim 6.3 \mathrm{~d}$ for BAL 090100001 [35], 9.8 d for PG 1219+534 (Green et al. 2013, in prep), 39.2 d for 


\section{Ageing Low Mass Stars: From Red Giants to White Dwarfs}

KPD 1943+4058 [36], and 27.5 d for KIC 10139564 [37]. Accumulated Kepler data suggest that the rotation rate could even be much smaller in some cases, leading to the overall impression that isolated $\mathrm{sdB}$ stars are in general very slow rotators considering their compact nature. This is qualitatively in agreement with the finding from spectroscopy that most isolated or well detached sdB stars have very low $V \sin i$ and therefore rotate slowly at their surface [38]. If confirmed, such low rotation rates for isolated $\mathrm{sdB}$ stars could be a key element for deciding which formation channel prevails to produce these objects. The helium white dwarf merger proposed as a potentially dominant channel to form isolated sdB stars [39] could have major difficulties in this context, unless a mechanism to evacuate the huge angular momentum stored in the merger can be found in order to form very slowly rotating sdB stars. Isolated sdB stars may then have followed a more classical evolution through the red giant phase, in which case their rotation somewhat represents the end product of the evolution of angular momentum during the red giant branch (see the contribution of B. Mosser in these proceedings).

\section{SUMMARY AND CONCLUSION}

Quantitative asteroseismology of hot B subdwarf stars has been an active field for more than 10 years, thanks to essential observational efforts to characterize the pulsations in these stars, and to dedicated theoretical and numerical developments to exploit these observations. It permitted precise determinations of key fundamental parameters for 15 pulsating sdB stars, so far, allowing for detailed investigations of individual objects as well as to give new keys to understand the evolution and formation of these objects. The recent advent of space observations with CoRoT and Kepler has further increased the potential of this technique with the possibility to use the deep sounding $g$-modes to probe the stars down to their central core where helium burning takes place.

Among the many outcomes of sdB asteroseismology, probing the internal stratification inside these stars is one of the possibilities that cannot be achieved through other known techniques. In particular, the mass of the H-rich envelope, which is the residual of the former red giant envelope, can be inferred. The size of the central mixed core and the amount of helium left in it can also be estimated with the $g$-modes. This gives us important information to study physical ingredients of stellar models that are not well understood (convection and overshooting, partial mixing zones, extra mixing).

Asteroseismology is also the only tool that can potentially reveal the internal rotation of stars. For hot B subdwarfs, we are just at the beginning of fully exploiting this possibility which can help to address several problems. For sdB stars in close binaries, rotation should be linked to the tidal interaction between the star and its companion. In this context asteroseismic constraints will bring crucial tests for tidal synchronization theories. In contrast, isolated $\mathrm{sdB}$ stars should be in rotation states that derive from their secular evolution. Hence, learning about this rotation by asteroseismic means will be a key factor to identify the main formation channels of lonely sdB stars. For those that evolved through the red giant branch, their internal rotation should be the end product of the angular momentum evolution during that phase.

Finally, non-adiabatic asteroseismology of sdB stars has received little attention in this revue, but its potential is also extremely interesting (see [40]), as the driving mechanism strongly depends on radiative levitation and atomic opacities. Consequently, a number of "non-adiabatic observables" exists that could in principle bring new constraints on microscopic diffusion mechanisms and their potential competitors (e.g., stellar winds, turbulent mixing, thermohaline convection [41, 42]).

\section{References}

[1] Heber, U. ARA\&A, 47, (2009) 211

[2] Fontaine, G., Brassard, P., Charpinet, S., et al. A\&A, 539, (2012) 12

[3] Saffer, R.A., Bergeron, P., Koester, D., \& Liebert, J. ApJ, 432, (1994) 351

[4] Kilkenny, D., Koen, C., O’Donoghue, D., \& Stobie, R. S. MNRAS, 285, (1997) 640 
[5] Green, E.M. et al. ApJ, 583, (2003) L31

[6] Schuh, S. et al. ASPC, 334, (2005) 530

[7] Charpinet, S., Fontaine, G., Brassard, P., et al. ApJ, 483, (1997) 123

[8] Fontaine, G., Brassard, P., Charpinet, S., et al. ApJ, 597, (2003) 518

[9] Billères, M., Fontaine, G., Brassard, P., Liebert, J. ApJ, 578, (2002) 515

[10] Østensen, R.H., Oreiro, R., Solheim, J.-E., Heber, U., et al. A\&A, 513, (2010) 6

[11] Kilkenny, D., Reed, M.D., O’Donoghue, D., Kawaler, S.D., et al. MNRAS, 345, (2003) 834

[12] Charpinet, S., Fontaine, G., Brassard, P., Green, E.M., \& Chayer, P. A\&A, 437, (2005) 575

[13] Jeffery, C.S., Dhillon, V.S., Marsh, T.R., \& Ramachandran, B. MNRAS, 352, (2004) 699

[14] Randall, S.K., Fontaine, G., Brassard, P., \& Bergeron, P. ApJS, 161, (2005) 456

[15] Charpinet, S., Fontaine, G., Brassard, P., Randall, S.K., et al. ASPC, 392, (2008) 297

[16] Telting, J.H., Geier, S., Østensen, R.H., Heber, U., et al. A\&A, 492, (2008) 815

[17] Randall, S.K., Fontaine, G., Brassard, P., \& Van Grootel, V. A\&A, 522, (2010) 48

[18] Charpinet, S., Green, E.M., Baglin, A., Van Grootel, V., et al A\&A, 516, (2010) L6

[19] Østensen, R.H., Silvotti, R., Charpinet, S., Oreiro, R., et al. MNRAS, 409, (2010) 1470

[20] Østensen, R.H., Silvotti, R., Charpinet, S., Oreiro, R., et al. MNRAS, 414, (2011) 2860

[21] Charpinet, S., Fontaine, G., Brassard, P., Dorman, Ben ApJS, 131, (2000) 223

[22] Charpinet, S., Fontaine, G., Brassard, P., Dorman, Ben ApJS, 139, (2002) 487

[23] Charpinet, S., Fontaine, G., Brassard, P., Dorman, Ben ApJS, 140, (2002) 469

[24] Telting, J.H., Østensen, R.H., Baran, A.S., Bloemen, S., et al. A\&A, 544, (2012) 1

[25] Reed, M.D., Baran, A., Quint, A.C., Kawaler, S. D., et al. MNRAS, 414, (2011) 2885

[26] Brassard, P., Fontaine, G., Billères, M., Charpinet, S., et al. ApJ, 563, (2001) 1013

[27] Charpinet, S., Fontaine, G., \& Brassard, P. PASP, 113, (2001) 775

[28] Charpinet, S., Van Grootel, V., Reese, D., Fontaine, G., et al. A\&A, 489, (2008) 377

[29] Brassard, P. \& Fontaine, G. ASPC, 392, (2008) 261

[30] Van Grootel, V., Charpinet, S., Fontaine, G., Green, E.M., Brassard, P. A\&A, 524, (2010) 63

[31] Van Grootel, V., Charpinet, S., Fontaine, G., Brassard, P., et al. ApJ, 718, (2010) L97

[32] Charpinet, S., Van Grootel, V., Fontaine, G., Green, E.M., et al. A\&A, 530, (2011) 3

[33] Pablo, H., Kawaler, S.D., Reed, M.D., Bloemen, S., et al. MNRAS, 422, (2012) 1343

[34] Pablo, H., Kawaler, S.D., \& Green, E.M. ApJ, 740, (2011) L47

[35] Baran, A., Oreiro, R., Pigulski, A., Pérez Hernández, F., et al. MNRAS, 392, (2009) 1092

[36] Charpinet, S., Fontaine, G., Brassard, P., Green, E.M., et al. Nature, 480, (2011) 496

[37] Baran, A.S., Reed, M.D., Stello, D., Østensen, R.H., et al. MNRAS, 424, (2012) 2686

[38] Geier, S. \& Heber, U. A\&A, 534, (2012) 149

[39] Han, Z., Podsiadlowski, Ph., Maxted, P.F.L., \& Marsh, T.R. MNRAS, 341, (2003) 669

[40] Charpinet, S.; Fontaine, G.; Brassard, P. A\&A, 493, (2009) 595

[41] Hu, Haili, Tout, C.A., Glebbeek, E., \& Dupret, M.-A. MNRAS, 418, (2011) 195

[42] Théado, S., Vauclair, S., Alecian, G., \& Le Blanc, F. ApJ, 704, (2009) 1262 